\title{
Translator's Acknowledgment
}

I should like to take the opportunity to acknowledge the generous assistance of Dr. Rick Graebner of the Music Department of Southampton University, who read through the entire manuscript and made countless suggestions and improvements, as well as contributing a number of footnotes.

R.L. 

SOUND FIGURES 
